\title{
Performance Analysis of Compressive Sensing based LS and MMSE Channel Estimation Algorithm
}

\author{
Ami Munshi, and Srija Unnikrishnan
}

\begin{abstract}
In this paper, we have developed and implemented Minimum Mean Square Channel Estimation with Compressive Sensing (MMSE-CS) algorithm in MIMO-OFDM systems. The performance of this algorithm is analyzed by comparing it with Least Square channel estimation with compressive sensing (LSCS), Least Square (LS) and Minimum Mean Square Estimation (MMSE) algorithms. It is observed that the performance of MMSE-CS in terms of Bit Error Rate (BER) metric is definitely better than LS-CS and LS algorithms and it is at par with MMSE algorithm. Moreover the role of compressive sensing theory in channel estimation is accentuated by the fact that in MMSE-CS algorithm only a very small number of channel coefficients are sensed to recreate the transmitted data faithfully as compared to MMSE algorithm.
\end{abstract}

Index Terms-Compressive sensing, LS, MMSE, channel estimation, MIMO, OFDM.

\section{INTRODUCTION}

MIMO-OFDM technology offers high spectral efficiency, high reliability, high data rate, mitigation of multipath fading effect, etc. Hence MIMO-OFDM technology has become the basis of Long Term Evolution (LTE) systems and wireless broadband communication systems [1-5]. Faithful and speedy recovery of the transmitted signal at the receiver side with minimum overhead in terms estimating the Channel State Information (CSI) is very crucial in any communication system. Abundant amount of work has already been done in exploring classical channel estimation techniques such as Least Square (LS) and Bayesian channel estimation techniques like Minimum Mean Square (MMSE). LS channel estimation technique is popular due to its simplicity in implementation. Also, it does not require any prior information of channel statistics. Although MMSE channel estimation requires prior information of the channel statistics and is more complex than the LS channel estimation technique, its performance in terms

Manuscript received June 12, 2020; revised January 14, 2021. Date of publication February 4, 2021. Date of current version February 4, 2021. The associate editor prof. Gordan Šišul has been coordinating the review of this manuscript and approved it for publication.

A. Munshi is with the Mukesh Patel School of Technology Management and Engineering, NMIMS University, Mumbai, India, 400056 (e-mail: ami.munshi@nmims.edu). S. Unnikrishnan is with the Fr Conceicao Rodrigues College of Engineering, Mumbai, India. (srija@ fragnel.edu.in).

Digital Object Identifier (DOI): 10.24138/jcomss.v17i1.1084 of metrics like Bit Error Rate (BER) and Mean Square Error (MSE) is much better than LS channel estimation technique [610]. Based on the theory that the transmission channel is sparse with only few major channel coefficients, compressive sensing based channel estimation algorithms are also gaining popularity [11-18]. As claimed by the compressive sensing theory, if the signal is sparse in its known basis, then fewer measurements of the signal may be needed to represent the signal in its compressed form [19-21]. Moreover, an appropriate recovery algorithm will be able to recreate the original signal from its compressed structure. In [22], LS-CS channel estimation technique is implemented in SISO and MIMO OFDM system which takes into account sparsity of the channel and explores the effectiveness of compressive sensing theory in channel estimation . In [23], the effect of varying Fast Fourier Transform (FFT) size in OFDM on the performance of LS-CS channel estimation algorithm is observed. In [24], performance of LS-CS is analyzed based on the recovery of transmitted audio signal over a sparse noisy channel. It is observed that with LSCS channel estimation technique, an appropriate reconstruction of transmitted data is possible at the receiver by just sensing a few channel coefficients in a very noisy channel, by selecting the optimum FFT size.

In this paper, we have developed and implemented MMSECS channel estimation algorithm. We have compared the performance of MMSE-CS algorithm with LS-CS algorithm implemented in [22] and its performance to recover audio data was analyzed in [24]. To accentuate the importance of compressive sensing in channel estimation, we have also compared the performance of MMSE-CS in terms of BER with classical LS and MMSE channel estimation algorithms.

In section II we briefly describe the design and implementation of LS-CS channel estimation algorithm that was implemented in [22], followed by implementation of MMSE-CS channel estimation algorithm. Section III gives the simulation results of LS, MMSE, LS-CS and MMSE-CS channel estimation techniques and its analysis, followed by conclusion in section IV.

\section{SYSTEM MODEL}

\section{A. LS and MMSE Channel Estimation Algorithms}

When the information signal is transmitted from transmitter 
to the receiver, it gets distorted due to channel characteristics. With the aid of an appropriate channel estimation algorithm, it is possible to recover the transmitted signal faithfully at the receiver with minimum error. In pilot aided channel estimation techniques, the channel characteristics or the CSI is estimated by transmitting a pilot signal which is also know to the receiver. LS and MMSE techniques are the most widely used and popular techniques to estimate the CSI [2],[25],[26] .

Consider the following equation

$$
Y=X H+V
$$

here $Y$ is the received signal vector corresponding to the transmitted pilot signal vector $X, \mathrm{CSI}$ is denoted by $H$ and $V$ is the noise vector. Then according to the LS channel estimation technique, the estimate of channel $H$, denoted by $\widehat{H}_{L S}$ is given by

$$
\widehat{H}_{L S}=Y X^{T}\left(X X^{T}\right)^{-1}
$$

This estimated CSI is further used to reconstruct the transmitted signal at the receiver side. As mentioned in [2], for MMSE channel estimation technique, the unknown channel statistics is estimated based on prior knowledge obtained from LS estimation as

$$
\widehat{H}_{M M S E}=W \widehat{H}_{L S}
$$

where $W$ is known as the weight matrix given by

$$
W=R_{H_{L S} \widehat{H}_{L S}} R_{\widehat{H}_{L S} \widehat{H}_{L S}}
$$

where $R_{H_{L S} \widehat{H}_{L S}}$ is the cross correlation matrix between the true channel vector and temporary channel estimated vector. $R_{\widehat{H}_{L S} \widehat{H}_{L S}}$ is the autocorrelation matrix.

\section{B. Compressive Sensing Theorem in Channel Estimation Algorithm}

Consider a signal of length $N$, which is sparse with $M$ significant channel coefficients, where $M \ll N$. According to compressive sensing theory, it is possible to reconstruct the original signal back even if only $K$ coefficients out of the total $N$ coefficients are sensed randomly by using appropriate measurement matrix and reconstruction algorithm [21],[27-30]. Here $K \ll N$ and is given by

$$
K \geq C M \ln \left(\frac{N}{M}\right)
$$

where $C>0$ is a constant.

Bernoulli matrix or Gaussian random matrix can be taken as measurement matrix $A$ of size $K X N$. Hence the compressive sensing problem could be stated in two parts where first we have to develop a good measurement matrix to sense the sparse signal and then use an appropriate recovery algorithm, to reconstruct the original signal.

It is observed that classical channel estimation techniques such as LS and MMSE, together with multicarrier modulation systems can considerably enhance the system performance [7],[31]. Taking into account sparsity of the channel with only few significant channel coefficients, we can apply the compressive sensing theory in sparse channel estimation, thereby reducing the percentage of total channel coefficients sensed which in turn reduce the mathematical complexity and processing time required to recover all the channel coefficients. In the next subsection, implementation of LS-CS and MMSECS channel estimation algorithms is detailed.

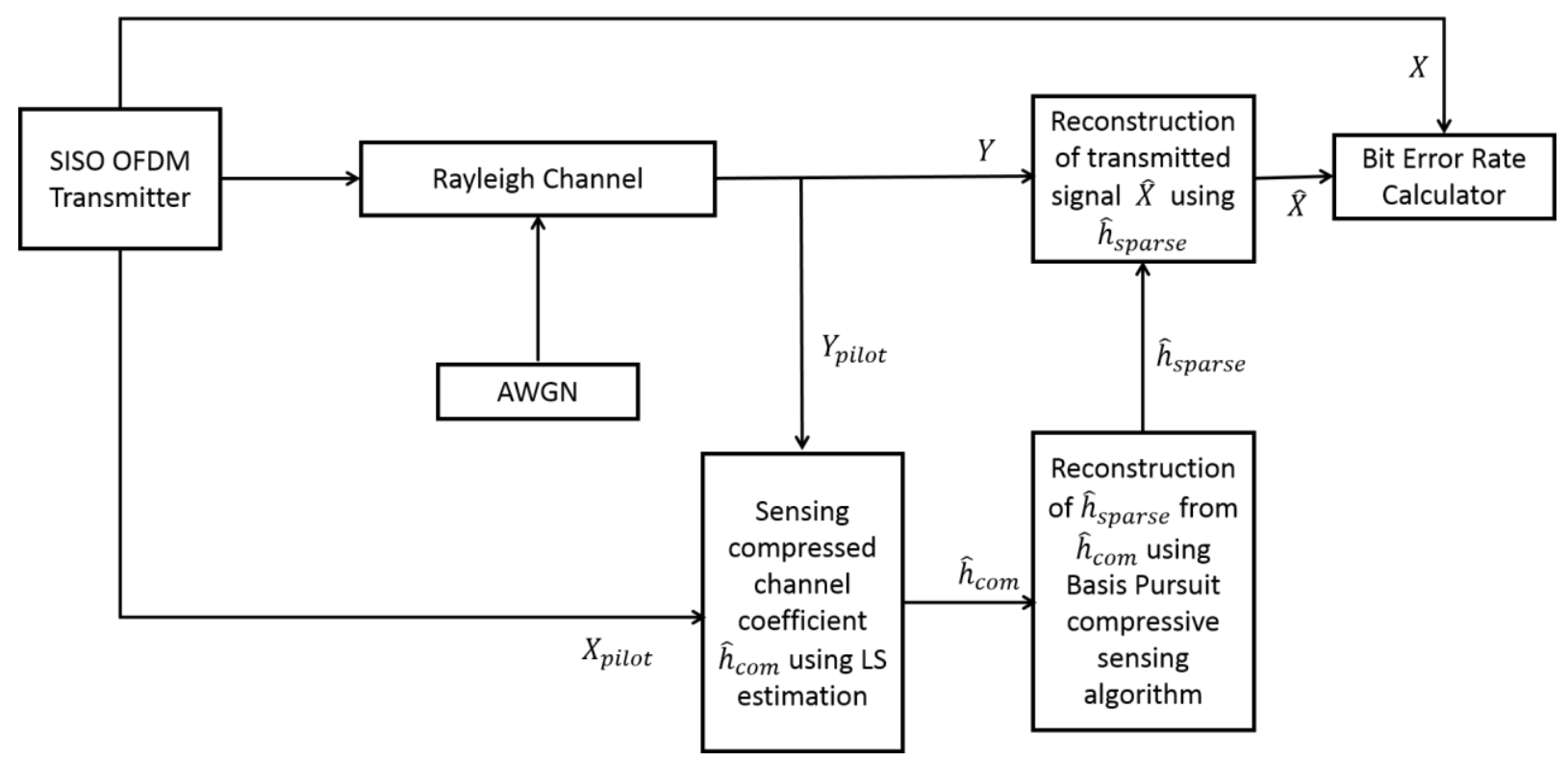

Fig. 1. LS-CS Channel Estimation Algorithm Block Diagram 


\section{Design and Implementation of LS-CS estimation Technique}

Fig. 1. shows the block diagram of LS-CS estimation technique which was implemented in [22] and Table I shows its algorithm.

The channel model considered in this implementation follows Rayleigh's distribution. The presented Clarke's model given in [32] is made sparse using a post processing algorithm. A block of pilot vector $\mathrm{X}$ is transmitted to obtain the received vector $Y$ in SISO-OFDM system. The received vector is given by equation (6)

$$
Y_{\text {pilot }}=\left(A h_{\text {sparse }}\right) * X_{\text {pilot }}+V
$$

here $A$ is a $K X N$ Gaussian random matrix, $h_{\text {sparse }}$ is the sparse channel coefficient vector and $V$ is the noise vector. Here number of rows, $K$ of the matrix $A$ stands for the number of channel coefficients sensed and is calculated using equation (5) and $N$ is the total number of channel coefficients in the given channel.

Let $h_{\text {com }}=A h_{\text {sparse }}$, be the compressed channel vector then
TABLE I

LS-CS CHANNEL ESTIMATION ALGORITHM

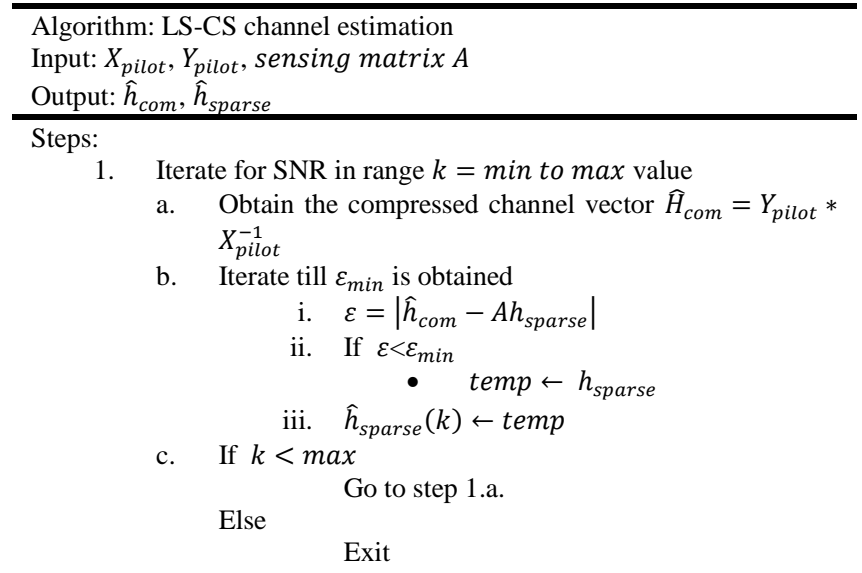

$\hat{h}_{\text {sparse }}$ is then used to reconstruct the transmitted data. The same logic can be further extended to estimate the channels in MIMO-OFDM systems.

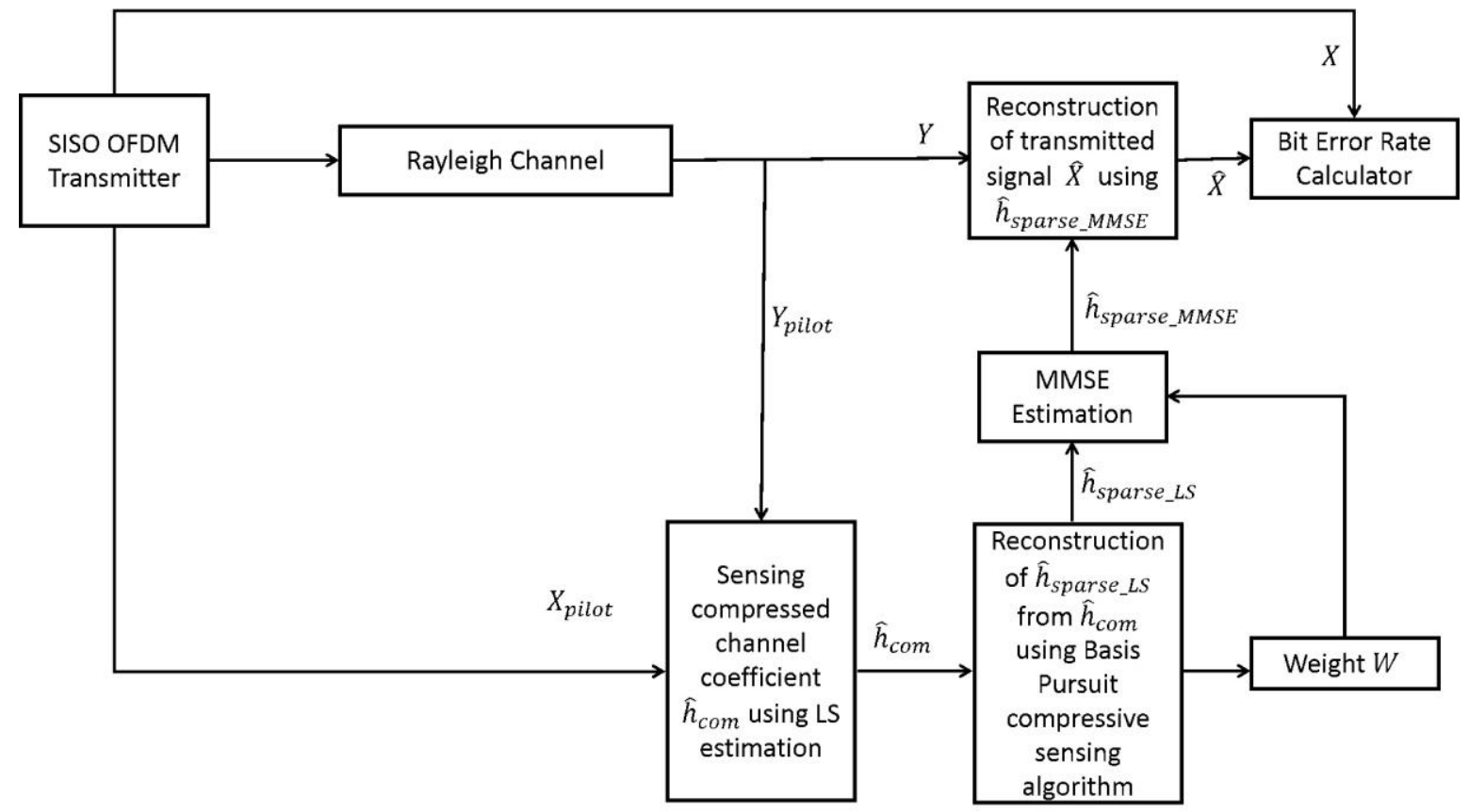

Fig. 2. MMSE-CS Channel Estimation Algorithm Block Diagram

equation (6) can be written as follows

$$
Y_{\text {pilot }}=h_{\text {com }} * X_{\text {pilot }}+V
$$

Here the compressed channel vector $h_{\text {com }}$ has only non-zero major channel coefficients. $h_{\text {com }}$ is estimated using LS estimation technique given by equation (2) to obtain $\hat{h}_{\text {com }}$. The estimate of sparse channel $\hat{h}_{\text {sparse }}$ is then obtained using from $\hat{h}_{\text {com }}$ using $l 1$ magic Basis Pursuit algorithm.

According to this algorithm, we obtain $\hat{h}_{\text {sparse }}$ such that error of $\left|\hat{h}_{\text {com }}-\hat{h}_{\text {sparse }}\right|$ is minimum. The estimated channel

\section{Design and Implementation of MMSE-CS Channel Estimation Algorithm}

In this section, we have implemented compressive sensing algorithm in MMSE channel estimation. We have constructed a sparse channel model using Clarke's prototype of Rayleigh channel. The compressed channel vector $h_{c o m}$ of equation (7) is obtained by using equation (2) and $\widehat{h}_{\text {sparse_LS }}$ is obtained using $l 1$ magic algorithm as done in LS-CS implementation. Then we obtain $\widehat{h}_{\text {sparse_MMSE }}$, which is the estimated channel using MMSE-CS technique, derived from $\widehat{h}_{\text {sparse_LS }}$ which is estimated using LS-CS technique. 
Hence,

$$
\widehat{h}_{\text {Sparse_MMSE }}=W * \widehat{h}_{\text {Sparse_LS }},
$$

where $W$ is the weight matrixgiven by equation (4). For MMSE-CS, the corresponding weight matrix $\mathrm{W}$ will be,

$$
\begin{aligned}
W=\left(R_{\left.h_{\text {sparse }} \widehat{h}_{\text {sparse_LS }}\right)}\right) \\
*\left(R_{\left.\widehat{h}_{\text {sparse_LS }} \widehat{h}_{\text {sparse } \_L S}\right),}\right.
\end{aligned}
$$

where $R_{h_{\text {Sparse }} \widehat{h}_{\text {Sparse_LS }}}$ is the cross-correlation matrix between true channel vector and estimated LS channel vector obtained.

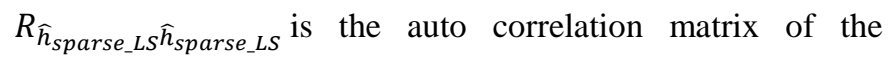
estimated LS channel vector.

Fig. 2. shows the block diagram of the MMSE-CS channel estimation technique implementation. It is seen from the block diagram that the process of MMSE-CS channel estimation is similar to LS-CS channel estimation; however, MMSE-CS obtains a better estimate of the channel due to the refined weight matrix. Here the channel coefficient vector using LS channel estimation $\widehat{h}_{\text {sparse_LS }}$ is estimated using $l 1$ magic algorithm. According to this algorithm, that value of $h_{\text {sparse }}$ which gives minimum value for $\left|\hat{h}_{\text {com }}-A h_{\text {sparse }}\right|$ is taken as $\widehat{h}_{\text {sparse_Ls }}$. Table II gives the algorithm of MMSE-CS channel estimation algorithm.

TABLE II

MMSE-CS CHANNEL ESTIMATION ALGORITHM

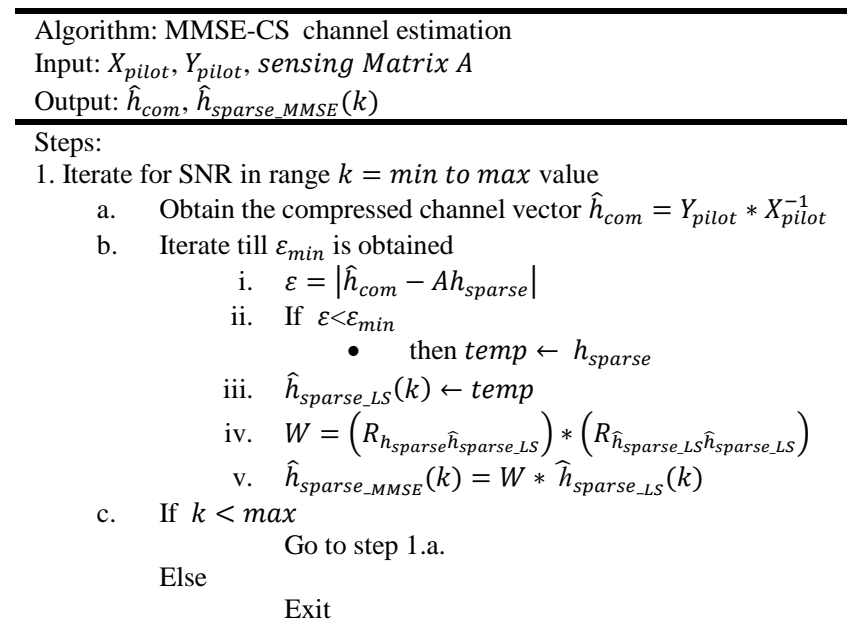

\section{SimUlation ResUlts AND Discussion}

In this section, the simulation results of LS, MMSE, LS-CS and MMSE-CS are presented. Performance of the aforementioned techniques are analyzed and compared taking into consideration various parameters like number of channel coefficients, sparsity of the channel, percentage of the total channel coefficients sensed and the FFT size.

\section{A. Performance Analysis of LS, LS-CS, MMSE, MMSE-CS in SISO OFDM Systems}

For this analysis, an image is transmitted over a sparse Rayleigh channel in SISO-OFDM system. The image is reconstructed at the receiver side by individually applying LS, MMSE, LS-CS and MMSE-CS channel estimation algorithms. The specifications used for the image transmission are given in Table III.

BER obtained for each channel estimation algorithm when the image is transmitted in a sparse channel with sparsity in the range of 6-11\% for various SNRs is specified in Table IV.

It is seen that all the channel estimation algorithms are capable of reconstructing the image at the receiver side faithfully with almost zero BER for all SNR equal to and above $5 \mathrm{~dB}$. For LS and MMSE all the 150 channel coefficients are sensed.

TABLE III

SYSTEM SPECIFICATIONS FOR SISO-OFDM SYSTEM

\begin{tabular}{|c|c|}
\hline Specification & Values \\
\hline Information size & $256^{*} 256^{*} 8$ bits \\
\hline Number of subcarriers & 64 \\
\hline $\begin{array}{c}\text { Total channel coefficients in } \\
\text { Rayleigh channel }(\mathrm{N})\end{array}$ & 150 \\
\hline Sparsity of channel $(\% \mathrm{M})$ & $6-11 \%$ \\
\hline $\begin{array}{c}\text { Percentage of channel } \\
\text { coefficients sensed (\% } \mathrm{K}) \text { in case } \\
\text { of LS-CS and MMSE-CS }\end{array}$ & $18-24 \%$ \\
\hline
\end{tabular}

TABLE IV

BER COMPARISON FOR LS, LS-CS, MMSE, MMSE-CS CHANNEL ESTIMATION ALGORITHM

\begin{tabular}{|c|c|c|c|c|}
\hline SNR & \multicolumn{5}{|c|}{ BER } \\
\hline & LS & LS-CS & MMSE & MMSE-CS \\
\hline 8 & 0 & 0 & 0 & 0 \\
\hline 5 & 0 & 0 & 0 & 0 \\
\hline 2 & 0.0018 & 0.0028 & 0.0015 & 0.0016 \\
\hline 0 & 0.0022 & 0.0031 & 0.0020 & 0.0021 \\
\hline
\end{tabular}

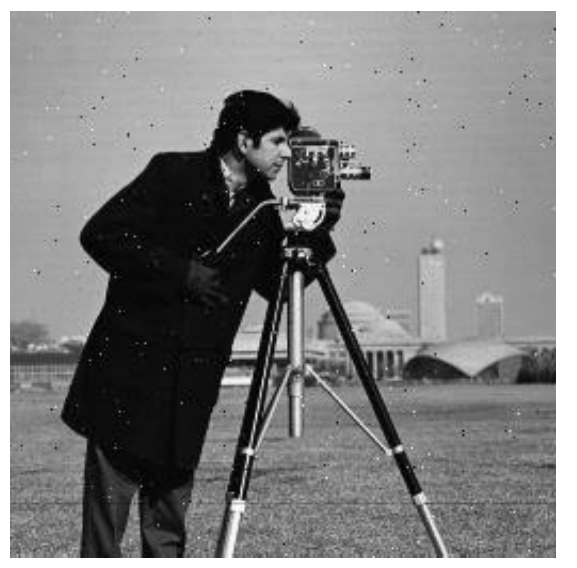

Fig. 3. Recovered image by employing LS estimation technique for $\mathrm{SNR}=2 \mathrm{~dB}$

However, for LS-CS and MMSE-CS, only $18-24 \%$ of the total 150 channel coefficients are sensed, thereby reducing the overhead of estimating all the channel coefficients. For $2 \mathrm{~dB}$ SNR, the BERs for MMSE and MMSE-CS are less than LS and LS-CS. Fig 3, 4, 5 and 6 show the reconstructed image using LS, LS-CS, MMSE and MMSE-CS channel estimation technique at $2 \mathrm{~dB}$ SNR respectively. 
It is observed that recovery of image using MMSE and MMSE-CS is better than LS and LS-CS. Nonetheless, the major advantage of MMSE-CS is that only $18-24 \%$ of the total 150 channel coefficients are sensed to recreate the image at the receiver.

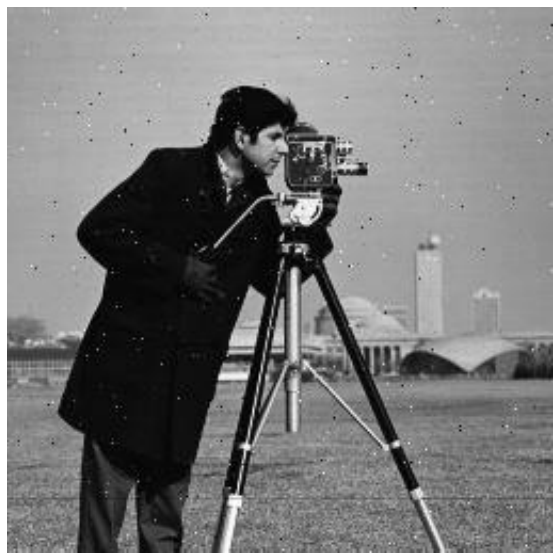

Fig. 4. Recovered image by employing LS-CS estimation technique for $\mathrm{SNR}=2 \mathrm{~dB}$

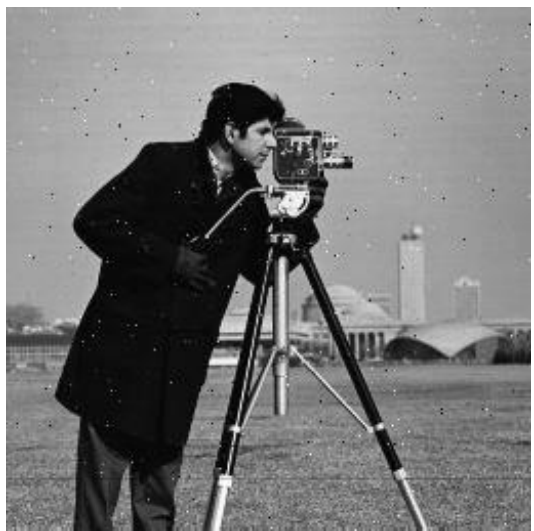

Fig. 5. Recovered image by employing MMSE estimation technique for $\mathrm{SNR}=2 \mathrm{~dB}$

\section{B. Performance Analysis of LS, LS-CS, MMSE, MMSE-CS in} $2 X 2$ MIMO OFDM Systems

Here, a scenario is considered where all the four channels of 2X2 MIMO have different sparsity levels. Here, along with the performance analysis of LS, MMSE, LS-CS and MMSE-CS channel estimation techniques, effect of sparsity on the performance with respect to BER metric is also analyzed.

At first, an image is transmitted over uncorrelated Rayleigh channels with different sparsity, at a given SNR of $2 \mathrm{~dB}$, in a 2X2 MIMO-OFDM system. The specifications used for the simulation are detailed in Table V. Table VI shows the values of BER obtained when the image through all the four channels is reconstructed using LS, LS-CS, MMSE and MMSE-CS channel estimation techniques.

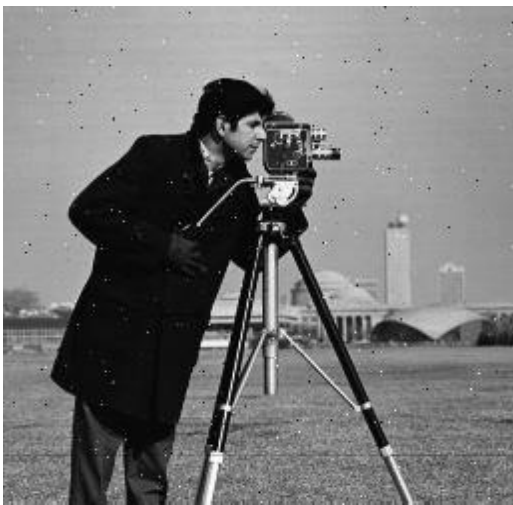

Fig. 6. Recovered image by employing MMSE-CS estimation technique for $\mathrm{SNR}=2 \mathrm{~dB}$

TABLE V

SYSTEM SPECIFICATIONS FOR MIMO OFDM

\begin{tabular}{|c|c|}
\hline Specification & Values \\
\hline System & $2 \times 2$ MIMO \\
\hline Information size & $2^{17}$ bits \\
\hline Number of subcarriers & 64 \\
\hline SNR & $2 \mathrm{~dB}$ \\
\hline $\begin{array}{c}\text { Total channel coefficients in } \\
\text { Rayleigh channel }(\mathrm{N})\end{array}$ & 150 \\
\hline
\end{tabular}

From Table VI, it is observed that the performance of MMSE and MMSE-CS is better than LS and LS-CS at any sparsity level. Moreover, we also deduce that the MMSE-CS gives a performance similar to MMSE, however by sensing just a small percentage of the total channel coefficients. For both MMSE-CS and LS-CS techniques, only some percentage of the total channel coefficients is sensed. For example, in case of channel 1, where the sparsity of the channel is only $7 \%$, only $19 \%$ of the total channel coefficients are sensed for both LSCS and MMSE-CS. However, MMSE-CS outperforms LS-CS by $48 \%$ in terms of BER. In addition, it is also noted that performance of all the channel estimation is better if the sparsity level is between $6-11 \%$ of the total channel coefficients.

Further a comparison of MMSE and MMSE-CS channel estimation is done in a $2 \mathrm{X} 2 \mathrm{MIMO}-\mathrm{OFDM}$ system with four channels of different sparsity level over a SNR range of 0 to $10 \mathrm{~dB}$. The specifications used for simulation are given in Table VII below. The sparsity level of each channel and the number of channel coefficients sensed are given in Table VIII.

TABLE VI

BER COMPARISON FOR LS, LS-CS, MMSE, MMSE-CS CHANNEL ESTIMATION ALGORITHM

\begin{tabular}{|c|c|c|c|c|c|c|c|}
\hline \multirow{2}{*}{$\begin{array}{l}\text { Channel } \\
\text { number }\end{array}$} & \multirow{2}{*}{$\begin{array}{l}\text { Sparsity of channel } \\
(\% \mathrm{M})\end{array}$} & \multirow{2}{*}{$\begin{array}{l}\text { Channel coefficients sensed } \\
(\% \mathrm{~K})\end{array}$} & \multicolumn{4}{|c|}{ BER } & \multirow{2}{*}{$\begin{array}{c}\text { \% improvement of } \\
\text { MMSE }\end{array}$} \\
\hline & & & LS & LS-CS & MMSE & MMSE-CS & \\
\hline 1 & $7 \%$ & $19 \%$ & 0.0421 & 0.0163 & 0.0082 & 0.00833 & $48 \%$ \\
\hline 2 & $12 \%$ & $26 \%$ & 0.1112 & 0.0911 & 0.0245 & 0.0249 & $72 \%$ \\
\hline 3 & $11 \%$ & $24 \%$ & 0.0147 & 0.0096 & 0.0027 & 0.0039 & $59 \%$ \\
\hline 4 & $15 \%$ & $28 \%$ & 0.0381 & 0.0254 & 0.0063 & 0.0067 & $73 \%$ \\
\hline
\end{tabular}




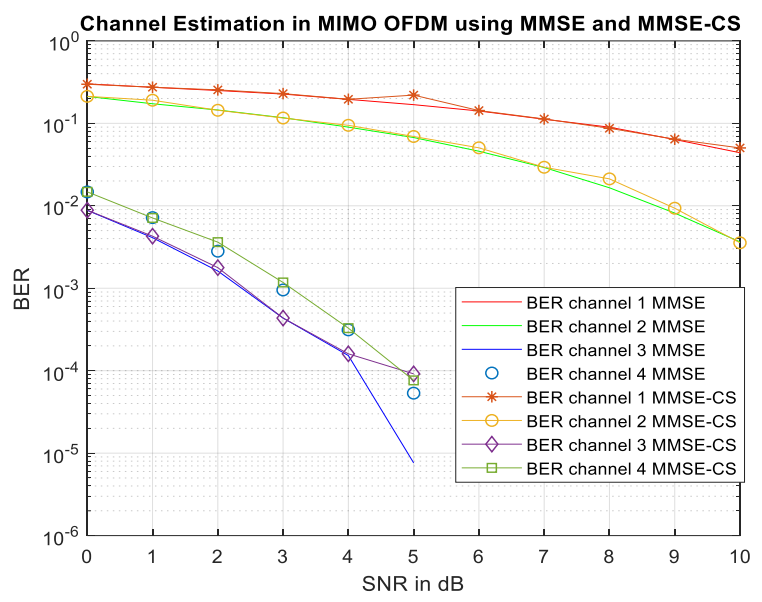

Fig. 7. BER plots for MMSE and MMSE-CS channel estimation technique in MIMO-OFDM

Fig. 7. portrays the SNR versus BER curve for all the four channels with different sparsity levels using MMSE and MMSE-CS channel estimation algorithm.

On analyzing Table VIII and Fig 7, the fact that sparser channel gives better performance in terms of BER established in [22] is affirmed. Channel 3 is the sparsest with only $10 \%$ significant non-zero channel coefficients. BER versus SNR curve for this channel is better than the other channels for both MMSE as well as MMSE-CS channel estimation technique. It is also observed that BER MMSE-CS is almost the same as MMSE. However, at $5 \mathrm{~dB}$ SNR, the BER for MMSE and MMSE-CS is $10^{-5.2}$ and $10^{-4.2}$ respectively. But at the same time, in MMSE-CS channel estimation technique, only $23 \%$ of the total channel coefficients are sensed to recover the transmitted data, whereas in MMSE channel estimation technique, all the channels coefficients are to be sensed to faithfully recreate the transmitted data.

\section{CONCLUSION}

The results obtained by implementing compressive sensing based MMSE-CS channel estimation algorithm are demonstrated in this paper. The result analysis indicates that the performance of MMSE-CS in terms of BER metric is definitely better than LS and LS-CS algorithms. It is also noted that MMSE-CS performance is at par with MMSE by sensing considerably lesser number of channel coefficients. The importance of compressive sensing theory in channel estimation is accentuated by the fact that LS-CS and MMSECS can faithfully recreate the transmitted data by sensing just a small number of channel coefficients, whereas in case of LS and MMSE, all the channel coefficients are sensed. In this paper it is also affirmed that sparser channel enhances the performance of channel estimation algorithm employing compressive sensing. Moreover, optimum performance of LS-CS and MMSE-CS channel estimation algorithm is attained when the channel sparsity is in the range of $6-11 \%$ by sensing just 18 $24 \%$ of the total channel coefficients.
TABLE VII

SYSTEM SPECIFICATION IN MIMO OFDM OVER SNR RANGE
\begin{tabular}{|c|c|}
\hline Specification & Values \\
\hline System & $2 \times 2$ MIMO \\
\hline Information size & $2^{17}$ bits \\
\hline Number of subcarriers & 64 \\
\hline SNR & $0: 10 \mathrm{~dB}$ \\
\hline $\begin{array}{c}\text { Total channel coefficients in } \\
\text { Rayleigh channel (N) }\end{array}$ & 60 \\
\hline
\end{tabular}

TABLE VIII

SPARSITY LEVEL AND CHANNEL COEFFICIENTS SENSED FOR FOUR CHANNELS IN 2X2 MIMO-OFDM SYSTEM

\begin{tabular}{|c|c|c|}
\hline Channel number & $\begin{array}{c}\text { Sparsity level } \\
(\% \mathrm{M})\end{array}$ & $\begin{array}{c}\text { Percentage of } \\
\text { channel coefficients } \\
\text { sensed } \\
(\% \mathrm{~K})\end{array}$ \\
\hline 1 & $16 \%$ & $30 \%$ \\
\hline 2 & $15 \%$ & $28 \%$ \\
\hline 3 & $10 \%$ & $23 \%$ \\
\hline 4 & $15 \%$ & $28 \%$ \\
\hline
\end{tabular}

\section{REFERENCES}

[1] A. K. Jagannatham, "Estimation for Wireless Communications -MIMO/ OFDM Cellular and Sensor Networks," NPTEL, 2016. https://onlinecourses.nptel.ac.in/noc16_ec01.

[2] C.-G. K. Yong Soo Cho, Jaekwon Kim, Won Young Yang, MIMO OFDM Wireless Communications with MATLAB. Singapore: IEEE Press, John Wiley \& Xons(Asia) Pte Ltd, 2010.

[3] H. Bölcskei, "MIMO-OFDM wireless systems: Basics, perspectives, and challenges," IEEE Wirel. Commun., vol. 13, no. 4, pp. 31-37, 2006, doi: 10.1109/MWC.2006.1678163.

[4] J. Jo and I. Sohn, "On the optimality of training signals for MMSE channel estimation in MIMO-OFDM systems," Eurasip J. Wirel. Commun. Netw., vol. 2015, no. 1, 2015, doi: 10.1186/s13638-015-0345-y.

[5] G. Ca, F. Georgescu, M. Nicolaescu, and A. Mocanu, "A Comparative Performance Analysis of MIMO-OFDM System over Different Fading Channels," IEEE Xplore, Electron. Comput. Artif. Intell. (ECAI), 2015 7th Int. Conf., pp. 1-4, 2015, doi: 10.1109/ECAI.2015.7301210.

[6] K. Liu and K. Xing, "Research of MMSE and LS channel estimation in OFDM systems," IEEE 2nd Int. Conf. Inf. Sci. Eng. ICISE2010 - Proc., vol. 1, no. 1, pp. 2308-2311, 2010, doi: 10.1109/ICISE.2010.5688562.

[7] A. Munshi and S. Unnikrishnan, "Modeling and Simulation of MIMOOFDM Systems with Classical and Bayesian Channel Estimation," in IEEE 2017 International Conference on Advances in Computing, Communication and Control (ICAC3), 2017, doi: 10.1109/ICAC3.2017.8318789.

[8] P. Sure and C. M. Bhuma, "A survey on OFDM channel estimation techniques based on denoising strategies," Eng. Sci. Technol. an Int. J., vol. 20, no. 2, pp. 629-636, 2017, doi: 10.1016/j.jestch.2016.09.011.

[9] K. Vidhya and R. S. Kumar, "Channel estimation techniques for OFDM systems," Int. Conf. Pattern Recognition, Informatics Med. Eng. PRIME 2012, vol. 1, no. 1, pp. 135-139, 2012, doi: 10.1109/ICPRIME.2012.6208301.

[10] F. A. P. De Figueiredo, F. S. Mathilde, F. P. Santos, F. A. C. M. Cardoso, and G. Fraidenraich, "On channel estimation for massive MIMO with pilot contamination and multipath fading channels," 2016 8th IEEE LatinAmerican Conf. Commun. LATINCOM 2016, vol. 19, no. 9, pp. 1660-1663, 2016, doi: 10.1109/LATINCOM.2016.7811581.

[11] J. Xiang, H. Yue, X. Yin, and L. Wang, "A New Smoothed L0 Regularization Approach for Sparse Signal Recovery," Math. Probl. Eng., vol. 2019, no. 3, 2019, doi: 10.1155/2019/1978154.

[12] Y. Peng, X. Yang, X. Zhang, W. Wang, and B. Wu, "Compressed MIMOOFDM channel estimation," Int. Conf. Commun. Technol. Proceedings, ICCT, pp. 1291-1294, 2010, doi: 10.1109/ICCT.2010.5689073. 
[13] I. Khan, M. Singh, and D. Singh, "Compressive sensing-based sparsity adaptive channel estimation for $5 \mathrm{G}$ massive MIMO systems," Appl. Sci., vol. 8, no. 5, 2018, doi: 10.3390/app8050754.

[14] W. Jiang, S. Zheng, Y. Zhou, F. Tong, and R. Kastner, "Exploiting time varying sparsity for underwater acoustic communication via dynamic compressed sensing," J. Acoust. Soc. Am., vol. 143, no. 6, pp. 3997-4007, 2018, doi: 10.1121/1.5042355.

[15] J. Cai, X. He, H. Wang, and R. Song, "Deterministic pilot design for structured sparse channel estimation in MISO systems," Wirel. Networks, vol. 26, no. 4, pp. 2609-2621, 2020, doi: 10.1007/s11276-019-02028-0.

[16] C. R. Berger, Z. Wang, J. Huang, and S. Zhou, "Application of compressive sensing to sparse channel estimation," IEEE Commun. Mag., vol. 48, no. 11, pp. 164-174, 2010, doi: 10.1109/MCOM.2010.5621984.

[17] W. Ding, F. Yang, C. Pan, L. Dai, and J. Song, "Compressive sensing based channel estimation for OFDM systems under long delay channels," IEEE Trans. Broadcast., vol. 60, no. 2, pp. 313-321, 2014, doi: 10.1109/TBC.2014.2315913.

[18] J. J. Mechery and M. Remadevi, "Compressive sensing based underwater channel estimation,” Procedia Comput. Sci., vol. 115, pp. 683-690, 2017, doi: 10.1016/j.procs.2017.09.155.

[19] R. G. Baraniuk, "Compressive Sensing [Lecture Notes]," IEEE Signal Process. Mag., vol. 24, no. July, pp. 118-121, 2007, doi: 10.1109/MSP.2007.4286571.

[20] D. L. Donoho, "Compressed sensing," IEEE Trans. Inf. Theory, vol. 52, no. 4, pp. 1289-1306, 2006, doi: Doi 10.1109/Tit.2006.871582.

[21] M. a Davenport, M. F. M. Duarte, Y. C. Y. Eldar, and G. Kutyniok, "Introduction to compressed sensing," Preprint, vol. 93, pp. 1-68, 2011, doi: 10.1287/mksc.20.3.244.9764

[22] A. Munshi and S. Unnikrishnan, "Compressive Sensing in Channel Estimation for SISO and MIMO OFDM Systems," in IEEE TENCON 2018 , 2018, doi: 10.1109/TENCON.2018.8650423

[23] A. Munshi and S. Unnikrishnan, "Performance analysis of a compressive sensing-based channel estimation algorithm in a noisy channel," IEIE Trans. Smart Process. Comput., vol. 8, no. 3, pp. 227-235, 2019, doi: 10.5573/IEIESPC.2019.8.3.227.

[24] A. Munshi and S. Unnikrishnan, "Recovery of compressed audio signal over a sparse channel in MIMO-OFDM systems," 2019 6th IEEE Int. Conf. Adv. Comput. Commun. Control. ICAC3 2019, pp. 1-6, 2019, doi: 10.1109/ICAC347590.2019.9036791.

[25] Y. S. Hussein, M. Y. Alias, and A. A. Abdulkafi, "On performance analysis of LS and MMSE for channel estimation in VLC systems," Proceeding - 2016 IEEE 12th Int. Colloq. Signal Process. its Appl. CSPA 2016, no. March, pp. 204-209, 2016, doi: 10.1109/CSPA.2016.7515832.

[26] V. Sharma and S. Kumar, "Recent developments in MIMO channel estimation techniques," 2012 2nd Int. Conf. Digit. Inf. Commun. Technol. its Appl. DICTAP 2012, pp. 1-6, 2012, doi: 10.1109/DICTAP.2012.6215335.

[27] Y. C. Eldar and G. Kutyniok, Compressed Sensing: Theory and Applications, First. Cambridge, UK: Cambridge, 2012.

[28] S. Foucart and H. Rauhut, A Mathematical Introduction to Compressive Sensing. London: Birkhauser, Springer, 2013.

[29] E. Candès and M. Wakin, "An introduction to compressed sensing," IEEE Signal Process. Mag., p. 21, 2008.

[30] R. G. Baraniuk, T. Goldstein, A. C. Sankaranarayanan, C. Studer, A Veeraraghavan, and M. B. Wakin, "Compressive video sensing: Algorithms, architectures, and applications," IEEE Signal Process. Mag., vol. 34, no. 1, pp. 52-66, 2017, doi: 10.1109/MSP.2016.2602099.

[31] A. Munshi and S. Unnikrishnan, "Design, Simulation, and Evaluation of," Int. J. Latest Technol. Eng. Manag. Appl. Sci., vol. 3, no. 1, pp. 12-26, 2017.

[32] Mathuranathan Viswanathan, "Simulation of Digital Communication Systems Using Matlab," Simul. Digit. Commun. Syst. Using Matlab, pp. 18, 2013, doi: 10.1002/ejoc.201200111.

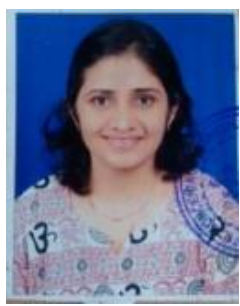

Ami Munshi is Assistant Professor in the Electronics and Telecommunication Department for the Mukesh Patel School of Technology Management and Engineering, NMIMS University, India. She has over 13 years of teaching experience at the UG level. She received her bachelor's and master's degrees in Engineering from the University of Mumbai, India. Currently, she is pursuing a $\mathrm{PhD}$ from Fr. Conceicao Rodrigues College of Engineering, University of Mumbai, under the guidance of Dr. Srija Unnikrishnan. Her research areas of interest include MIMO-OFDM, Channel Estimation, Compressive Sensing, Image Processing and Compression (image and audio).

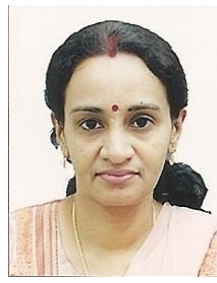

Srija Unnikrishnan is Principal at Fr. Conceicao Rodrigues College of Engineering, affiliated with the University of Mumbai, India. She has over 35 years of teaching experience at the UG and PG levels. She received her bachelor's degree in Engineering from the University of Kerala, a master's degree from Osmania University, and a PhD from the University of Mumbai. Her broad areas of interest are Mobile Communications and Signal Processing. 